\title{
Photocontrolled Reversible Dimensional Changes of Microstructured Photochromic Polymers
}

\author{
Despina Fragouli, Roberto Cingolani and Athanassia Athanassiou \\ Center for Biomolecular Nanotechnologies @UNILE, \\ Istituto Italiano di Tecnologia \\ Italy
}

\section{Introduction}

Stimuli-responsive polymeric materials are able to change their chemistry and their conformation upon an external signal. The external signal may be derived from a change in temperature, chemical composition or applied mechanical force of the specific material, or can be triggered externally with exposure to an electric or magnetic field or to light irradiation. In this respect, a photochromic substance is a stimuli responsive material which is characterized by its ability to alternate between two different chemical forms having different absorption spectra, in response to light irradiation of appropriate wavelengths (Brown 1971). Due to this important property, a significant amount of effort has been devoted to the formation of polymeric materials functionalized with photochromic molecules for the creation of photosensitive "smart material" systems, that change reversibly their physical and chemical properties by the use of light. The corresponding reversible effects of the molecules such as dipole moment, surface energy, refractive index, and volume are preserved in the polymer matrix, and have numerous promising applications in devices for three-dimensional (3D) optical memories, (S. Kawata \& Y. Kawata 2000), in actuators ( $Y u$ et al 2003, Athanassiou et al 2005), in holographic or diffractive optics, (Fu et al 2005, Tong et al 2005) or in microfluidics, (Caprioli et al 2007, Walsh et al 2010) etc. Concerning microfluidic devices using photochromic plastic films, the transportation of fluids happens without the need for their molecules to be charged, as done in other studies (Mitchel 2001). This is achieved by gradually modifying the surface tension, and thus the wettability, by irradiating with increasing time along the direction of the fluid movement (Ichimura et al 2000). The gradual wettability changes are exclusively based on the photochemical modification of the embedded photochromic molecules caused by the photoisomerization process. In addition, in the case of the diffraction gratings the development was generally done by interference of different polarized laser beams, or by electric-field application, and the modification of their diffraction efficiency is connected with the changes of the refractive index of the photochromic molecules during this procedure (Yamamoto et al 2001, Fu et al 2005).

Here we present how the volume changes induced to the photochromic polymers by the photoisomerization of their embedded photochromic molecules, can improve significantly 
the performance of these two different type of applications, namely the microfluidic devices and the diffraction gratings. In both cases the lithographic technique used for the microstructuring of the photochromic doped plastic films is the soft molding. Concerning the microfluidics applications, the presented microstructured photochromic plastic films exhibit a significant improvement on the reversible wetting characteristics compared to those on the flat surfaces. This improvement is due to the combination of the changes in the surface polarity and thus in the wetting properties with the modified surface conformation, both provoked by the light induced changes of the photochromic molecules. Moreover, regarding the optical gratings, we present a different approach in where the control of their diffraction efficiency relies on the dimensional variations of the gratings upon laser irradiation. Following this approach, the efficiency of the gratings is significantly improved with respect to previous works.

Such findings open the way for the production of optically switchable gratings based on reversible dimensional changes, and can be of great importance in all-optical signal processing systems. Moreover, the ability to control the wettability of surfaces by microstructuring and to tune it by using photochromic molecules, permits the application of these lithographically formed structures to all-optically controlled switches capable of operating with tunable speed, and to microfluidic actuation.

\section{Materials and methods}

\subsection{Photochromic polymers and properties}

Photochromic doped polymer films were prepared by incorporating photochromic molecules into polymer matrices. In particular, solutions of the polyethylmethacrylate-comethylacrylate copolymer (PEMMA) (average molecular weight, $\mathrm{M}_{\mathrm{w}}=100,000$ ) (Aldrich) mixed with the photochromic molecule $1^{\prime}, 3^{\prime}$-dihydro-1', $3^{\prime}, 3^{\prime}$ - trimethyl- 6-nitrospiro[2H-1benzopyran-2,2'-(2H)-indole] or (6-NO $\mathrm{NO}_{2} \mathrm{BIPS}$ ) (Aldrich) (weight ratio 90/10 respectively or $95 / 5$ ) were prepared in toluene. Consequently a certain volume of this solution was spincoated onto a glass substrate.

The photochromic dopant 6- $\mathrm{NO}_{2}$ BIPS, belongs to the family of spiropyrans (SP) which have been extensively studied in the past decades (Görner 1998). Initially it has a 3D structure, and exists predominately in its non polar form (Figure 1). It is colorless, denoting that it is transparent in the visible range of the spectrum, but absorbs in the ultraviolet (UV). Upon irradiation with UV light, it is converted to its isomeric form, merocyanine (MC), through the photochemical cleavage of its carbon-oxygen $\left(\mathrm{C}_{\text {spiro- }}-\mathrm{O}\right)$ bond. The $\mathrm{MC}$ has a planar structure, it is colored and polar, and has a new absorption band in the visible range of the spectrum. MC can revert back to the SP form photochemically, using visible-light irradiation.

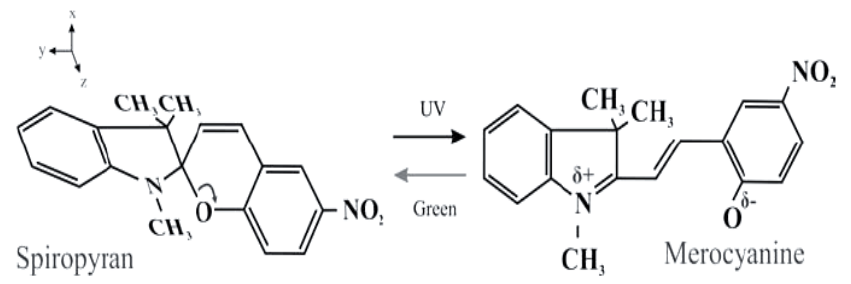

Fig. 1. The photochromic dopant spiropyran (SP) and the stable form of its isomer merocyanine $(\mathrm{MC})$. 
Briefly, UV and visible irradiation causes the reversible transformation of these chemical species, between two states (isomers) that have light absorption bands in characteristic spectral regions. This property is retained when the photochromic molecules are incorporated in polymer matrices, where they are homogeneously dispersed forming miscible systems. Specifically, the absorption properties of the photochromic polymer films prepared as described above change reversely upon UV-visible irradiation as shown at Figure 2. Initially the system is transparent at the visible range of the spectrum. Upon pulsed UV laser irradiation the SP is slowly converting to the MC isomer, fact indicated by the new absorption band in the visible region of the spectrum (ca. $565 \mathrm{~nm}$ ). The intensity of the peak increases with the number of UV pulses until a plateau is reached, which suggests that the photoisomerization is completed and that the system has reached the equilibrium. The subsequent irradiation with green laser light, causes the decrease of the intensity of the previously formed MC peak, while after a certain number of pulses the spectrum reaches its initial form, indicating that MC reverts fully to the SP isomer. These data confirm that under the irradiation conditions mentioned in the figure caption of Figure 2, the reversible properties of SP are retained in the host polymer matrix. Depending on the irradiation conditions and the weight percentage of the photochromic molecules in the polymer matrix (usually $\leq 10 \%$ ), it has been shown that typically about 4-10 irradiation cycles can be performed, while further irradiation causes the degradative photooxidation of the photochromic molecules, restricting thus the lifetime of the system (Athanassiou et al 2006c). Additionally, the degradative phenomena start to be evident usually after the third cycle. In order to exclude this parameter from the following study, results derived by the first three irradiation cycles are presented.

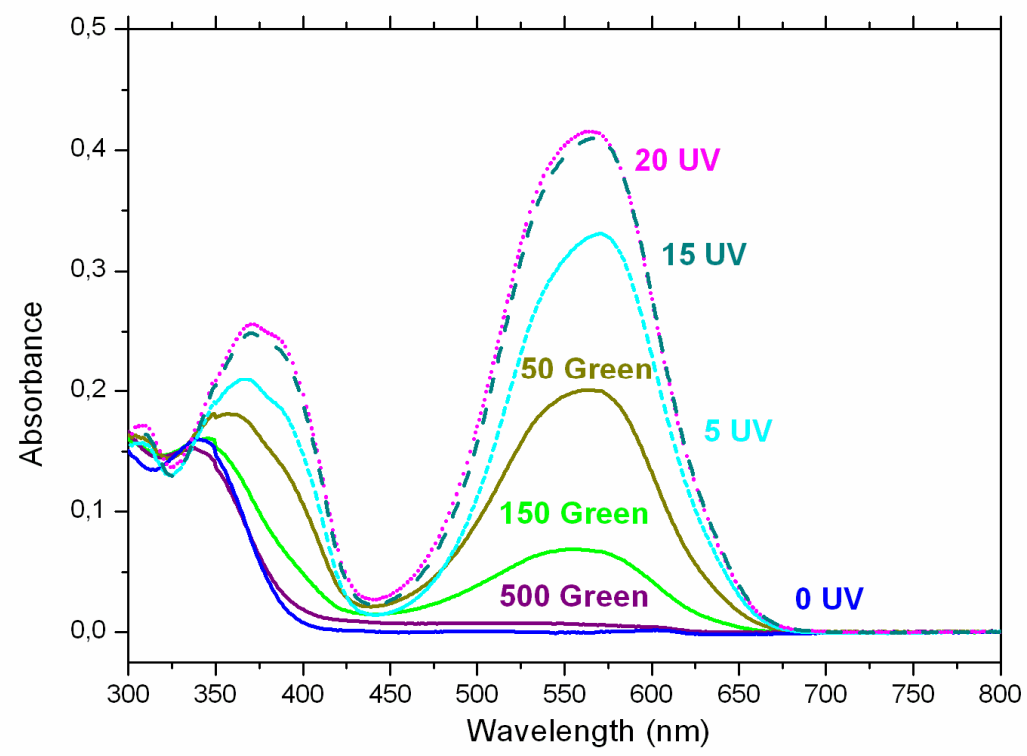

Fig. 2. Absorption spectra of the PEMMA/SP $10 \%$ wt upon UV and visible irradiation. For the specific study, the irradiation conditions used are: $\lambda_{\mathrm{UV}}=355 \mathrm{~nm}$, fluence $F_{\mathrm{UV}}=20 \mathrm{~mJ} \mathrm{~cm}^{-2}$, $\lambda_{\text {vis }}=532 \mathrm{~nm}, \mathrm{~F}_{\mathrm{vis}}=35 \mathrm{~mJ} \mathrm{~cm}^{-2}$. 
Except of the alteration in the optical properties, there are also other physical and chemical properties that change reversibly upon UV-visible irradiation even if the SP molecules are incorporated in the polymer matrix, such as dipole moment, surface energy, refractive index, and volume. Concerning the volume changes upon UV irradiation, occurs the formation of aggregates between different MC stereoisomers with zwitterionic character causing density fluctuations in the polymer matrix, reducing thus the MC partial molar volume. Consequently, takes place the short scale motion of the polymer chains in order to diminish the density fluctuations in the samples, and this leads to the macroscopic reduction of the dimensions of the matrix. This effect is reversible, since upon green irradiation, MC molecules return to the SP form, which does not form aggregates (Athanassiou et al. 2005)

\subsection{Substrates microstructuring: soft molding}

For the microstructuring of the photochromic plastic films it is used the soft molding lithography (SM). It is actually based on the conformal contact between the material to be patterned and an elastomeric replica of a master structure, and it combines soft and nanoimprint lithography, using elastomeric elements and exploiting the glass transition of organic compounds. Particularly, an elastomeric mold is placed onto a polymeric film applying the pressure of its own weight, and consequently is heated up above the films' glass transition temperature, $T_{g}$. The subsequent cooling down, below $T_{g}$, freezes the pattern into the polymer, and the replica is peeled off. The micropatterns formation is based on the capillarity effect that drives the polymer to penetrate into the recessed features of the elastomeric replica. The SM presents various advantages compared to the nanoimprint lithography. Specifically, since penetration of the polymer into recessed features of the replica is driven by capillarity effects, SM is only slightly affected by problems caused by difficult polymer transport. Moreover, it does not need any pressing setup to ensure contact between the mold and the polymer. Finally, SM does not present pattern shrinkage and distortion due to the solvents employed by other soft lithography techniques. (Pisignano et al 2004)

In Figure 3 it is represented the process followed for the SM. Initially, the original master structures are fabricated onto glass or Si by both photo- and electron-beam lithography. The realized masters are used as templates on which elastomeric replicas were realized using polydimethylsiloxane (PDMS, Sylgard 184, Dow Corning, Midland, MI) according to a standard replica molding procedure, and placed onto polymer films under their own weight. (Pisignano et al 2004)

In order to form the microstructured photochromic polymer substrates for the wettability study, elastomeric molds of PDMS having periods $\alpha=1.3,28.0$, and $180.0 \mu \mathrm{m}$ were placed onto flat spin-cast films. Then the system was heated at $50{ }^{\circ} \mathrm{C}$, a temperature higher than the $\mathrm{T}_{\mathrm{g}}$ of the PEMMA films $\left(\mathrm{T}_{\mathrm{g}}=48{ }^{\circ} \mathrm{C}\right)$. After the thermal cycle, the replica was easily peeled off from the photochromic polymer substrates, on which the patterns are transferred. For the preparation of the gratings on the films for the diffraction efficiency study, the substrates were placed on a hot plate and heated until they reach a temperature $\mathrm{T}_{\text {grating }}$ of $65^{\circ} \mathrm{C}$. Then an elastomeric mold with $\alpha=4 \mu \mathrm{m}$ was placed on the substrate for 10 min, resulting in the formation of the gratings. In both cases the SM procedure was carried out in nitrogen atmosphere to avoid the deterioration of the photochromic molecules upon heating. 


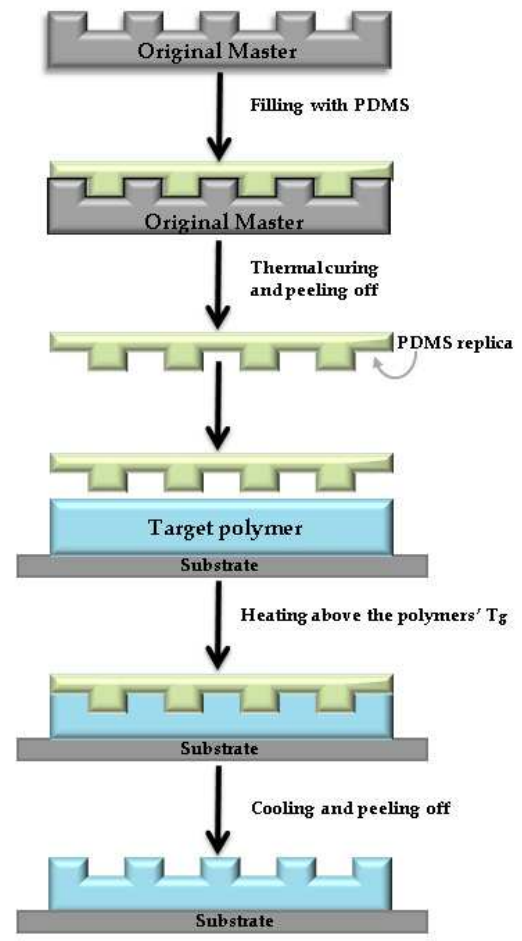

Fig. 3. Schematic diagram of the process of master replication and soft molding.

\section{Light induced wettability changes of patterned substrates}

\subsection{Introduction}

As abovementioned, the modification of the wetting characteristics of photochromic surfaces depends mainly on the photochemical processes which modify the surface tension and are caused by the UV-visible irradiation cycle. However, studies on patterned surfaces have demonstrated that the surface roughness affects significantly the wettability properties (Patankar 2003). Here it is studied the combined effect of the two aforesaid factors on the wettability properties of patterned photochromic polymeric surfaces. The wettability changes induced by photomechanical and photochemical changes are reversible upon UV-visible light irradiation, resulting in reversible changes of the wetting properties of the surfaces.

In particular, it is shown that the hydrophilicity of the photochromic polymeric surfaces is increased upon UV laser irradiation due to the polarity change caused by the photoisomerization, while the process is reversed upon green laser irradiation. The microstructuring of the surfaces enhances significantly the hydrophobicity of the system due to the increased surface roughness, and the light-induced wettability variations of the structured surfaces are enhanced by a factor of 3 compared to those on the flat surfaces. (Athanassiou et al 2006a, 2006b) In addition, by changing the topological parameters of the introduced pattern (e.g. by decreasing the period), are achieved higher differences in the surface wetting properties (Lygeraki et al 2008). 


\subsection{Results and discussion}

Figure 4, shows the apparent water contact angle (WCA) of a drop on a flat photochromic polymer surface before and after pulsed UV and green laser irradiation. The WCA on the flat substrate, $\theta_{Y}$, is formed when the liquid is in contact with a solid surface in static equilibrium with its vapor, and is determined by the Young's equation:

$$
\gamma_{\mathrm{LV}} \cos \theta_{\mathrm{Y}}=\gamma_{\mathrm{SV}}-\gamma_{\mathrm{SL}}
$$

where $\gamma_{\mathrm{LV}}, \gamma_{\mathrm{SV}}$ and $\gamma_{\mathrm{SL}}$ represent the interfacial tensions at the boundaries between the liquid (L), vapor (V), and solid (S).
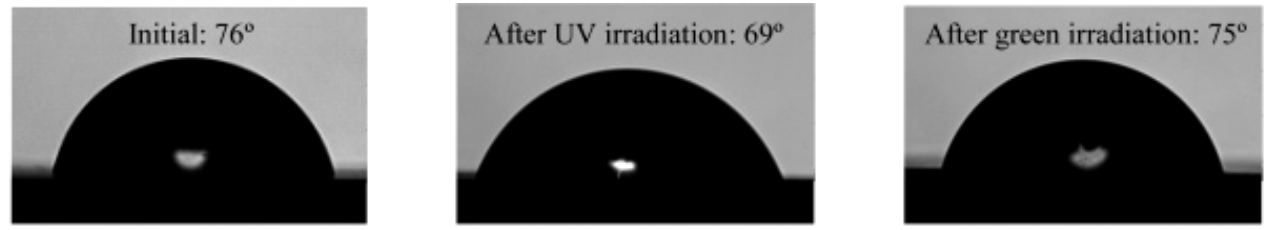

Fig. 4. WCA of a water drop of volume $3 \mu \mathrm{l}$ on flat photochromic polymer surface before

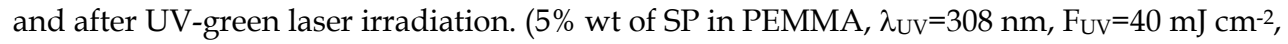
$\lambda_{\text {green }}=532 \mathrm{~nm}, \mathrm{~F}_{\text {green }}=45 \mathrm{~mJ} \mathrm{~cm}^{-2}$ ) (Athanassiou et al 2006a)

As shown, before any irradiation the surface is hydrophilic with a WCA of $76^{\circ}$. After irradiation with enough UV laser pulses so as to reach the complete photoisomerization of all the non polar SP molecules to the polar MC form (50 pulses), the surface becomes more hydrophilic, with a WCA of $69^{\circ}$. The subsequent green irradiation with 500 laser pulses causes the reversible phenomenon, which is the conversion of the MC isomer to the SP form, and the increase of the WCA until it reaches its initial value. The maximum WCA difference measured on numerous flat surfaces upon UV irradiation was $7^{\circ} \pm 1^{\circ}$.

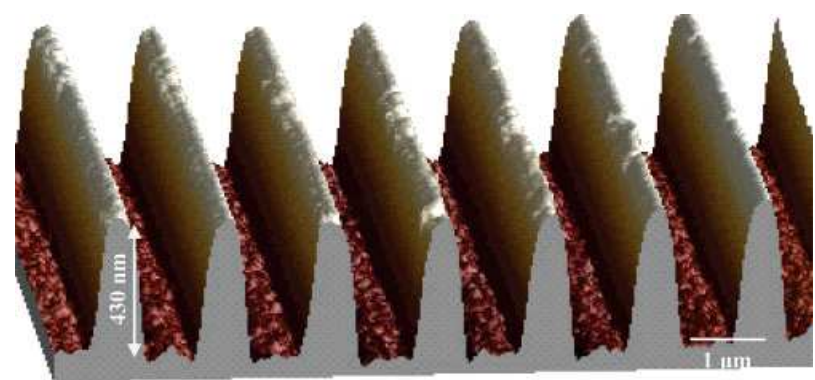

Fig. 5. Atomic force microscopy images of typical patterned surfaces of photochromic polymers. (Athanassiou et al 2006a)

In order to examine the effect of the photomechanical changes upon the same irradiation conditions, the aforementioned surfaces were microstructured using the SM technique. The replica used had a period $\alpha=1.3 \mu \mathrm{m}$, and by following the already described steps there were formed on the photochromic polymeric surface patterns of the same period (Figure 5). The WCA on the specific surface was found to be greatly affected by the patterning, showing an increased hydrophobicity with a value almost $30^{\circ}$ greater than that of the flat 
surfaces (Figure 6). Moreover, always in comparison with the flat surfaces, the light induced WCA changes due to the photoisomerization effect are enhanced by a factor of 3 , since the WCA change before and after UV irradiation is ca. $20^{\circ}$ (WCA change on the flat surface, ca. $7^{\circ}$ ). This guides to the conclusion that the microstructuring affects significantly the reversible photoinduced wettability changes of the surfaces.
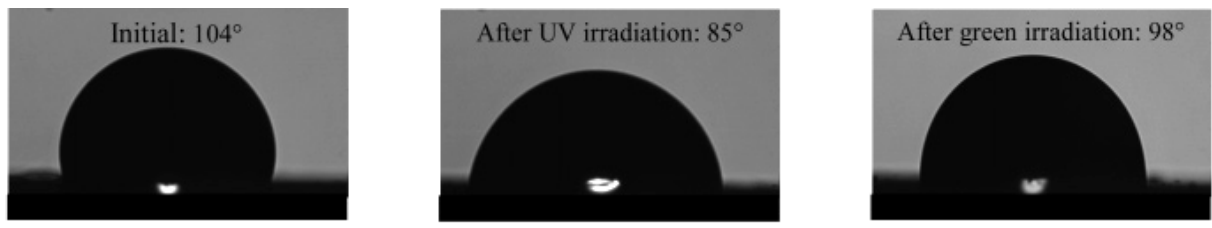

Fig. 6. WCA images obtained on patterned surfaces before and after laser irradiation. (5\% wt of SP in PEMMA, $\lambda_{\mathrm{UV}}=308 \mathrm{~nm}, \mathrm{~F}_{\mathrm{UV}}=40 \mathrm{~mJ} \mathrm{~cm}{ }^{-2}, \lambda_{\text {green }}=532 \mathrm{~nm}, \mathrm{~F}_{\text {green }}=45 \mathrm{~mJ} \mathrm{~cm}^{-2}$ )

(Athanassiou et al 2006a)

In order to explain the effect of roughness on the wetting characteristics of a surface, there are proposed two theories. The first is referred to as the Cassie-Baxter model (Cassie and Baxter 1944) (Figure 7a), and describes the wettability of rough surfaces, where only partial wetting may occur due to the trapping of air underneath the drop at the recessed regions of the surfaces. Since the drop is situated partially on air, the surface exhibits an enhanced hydrophobic behavior. The second one is the Wenzel model (Wenzel 1936), and it proposes that roughness increases the liquid-solid interfacial area, and thus hydrophilic surfaces $\left(\theta<90^{\circ}\right)$ become more hydrophilic, and hydrophobic $\left(\theta>90^{\circ}\right)$ more hydrophobic (Figure $\left.7 \mathrm{~b}\right)$.

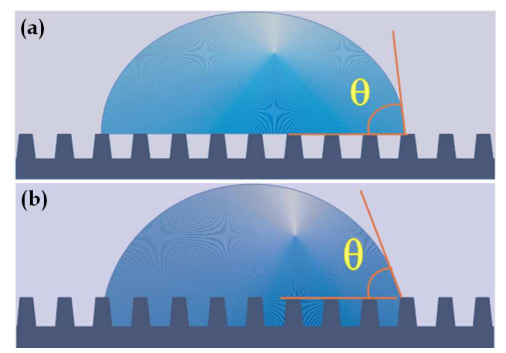

Fig. 7. Representation of a drop on a patterned surface, according to Cassie-Baxter model (a) and to Wenzel model (b).

In the presented cases the WCA of the drop on the flat surface is $76^{\circ}$ thus hydrophilic $\left(<90^{\circ}\right)$ and according to the Wenzel model the patterning should increase its hydrophilicity. However, the experimental results presented above show that the WCA after the patterning is significantly increased, reaching a maximum value of $104^{\circ}$, indicating that the surface became hydrophobic $\left(>90^{\circ}\right)$. Thus, the presented surfaces follow the Cassie-Baxter model, where the relation with the WCA of the flat surface $\left(\theta_{Y}\right)$ is given by the following equation:

$$
\cos \theta_{\mathrm{r}}=-1+\mathrm{f}_{\mathrm{s}}\left(1+\cos \theta_{\mathrm{Y}}\right)
$$


with $f_{s}$ the solid fraction of the surface in contact with the liquid. According to this equation, the WCA decreases when the $f_{s}$ is increasing. Using the WCA $\left(\theta_{Y}\right)$ measured on the flat surface and the one of the patterned in all three cases, before, after UV, and after green irradiation, the $f_{s}$ is calculated. The results show that the fraction of the patterned surface in contact with the liquid is increased after UV irradiation $\left(f_{s}=0.8\right)$ compared to the no irradiated sample $\left(f_{s}=0.6\right)$. AFM microscopy studies of the topological changes of the gratings after UV irradiation, showed that the average volume decrease of each nanoimprinted stripe is ca. $30 \mathrm{~nm}$. However, since the $f_{s}$ is higher, and the stripes of the pattern are narrower, it is concluded that the water drop penetrates deeper into the channels of the UV irradiated pattern (partial wetting-Figure $8 \mathrm{~b}$ ), decreasing thus the WCA value. After irradiation with green laser pulses the MC molecules return to their SP isomers, the stripes recover their previous volume, and thus the drop returns to its previous condition, demonstrating once more that the wetting behavior is greatly influenced by both, the changes in the surface polarity and the volume of the stripes (Athanassiou et al 2006a).

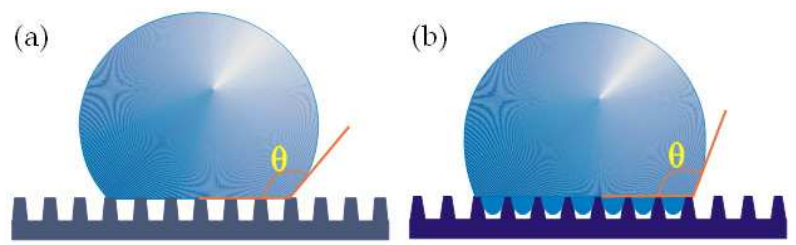

Fig. 8. Representation of a drop on a patterned surface, according to Cassie-Baxter model, before (a) and after (b) UV irradiation.

A further demonstration of the abovementioned statement is the study of the wetting properties of patterned surfaces with different topological parameters. In particular, the periods of the elastomeric replicas used for the formation of the patterns on the photochromic polymer surfaces (10\% wt of SP in PEMMA) were 1.3, 28.0, and $180.0 \mu \mathrm{m}$. Figure 9a demonstrates the WCA changes upon three UV-green irradiation cycles for each of the patterned substrates and the comparison with the flat surface. For comparison reasons it is presented also the WCA of the pure polymeric surface, without any addition of photochromic molecules, which is not affected by the irradiation as expected. As shown, the patterning of the surfaces increases the initial WCA in all three cases. However, the smaller the period of the pattern the more hydrophobic is its behavior compared to the flat surface. After UV irradiation, all substrates become more hydrophilic, but again the WCA difference from the initial one is greater at the pattern with the smaller period, and becomes smaller as the period is increasing (Figure $9 b$ ).

The observed difference between the various patterns cannot be attributed to the surface chemistry changes upon UV-green irradiation, since this is the same in all samples. Additionally, if this was happening, it should be observed the inverse phenomenon, thus the greater change for the pattern with the greater period, since in these cases the drops are in contact with a greater percentage of the solid surface, as seen from the calculated value of $f$, at Table 1 . Thus, the reported differences can be attributed to the volume shrinkage of the patterns upon UV irradiation. This reduction of the patterns volume affects much more the contact angle of the samples with patterns of smaller period, since the drop lies on a greater number of patterned features. Indeed it is calculated that the radius of the drop is 20 times 
larger than the size of each feature projected on the plane of the interface in the case of the patterns with a $180 \mu \mathrm{m}$ period, about 140 times greater in the case of the patterns with a 28 $\mu \mathrm{m}$ period, and finally more than 2950 times greater in the case of the patterns with a $1.3 \mu \mathrm{m}$ period. Accordingly, as shown at the table, for the pattern with period of $180 \mu \mathrm{m}$ there is a slight change of the $f$ upon UV-green irradiation. This is not the case for the smaller pattern where the change upon UV-green irradiation is almost $15 \%$, while for the pattern of period $28 \mu \mathrm{m}$ there is an intermediate change of ca. $6 \%$.

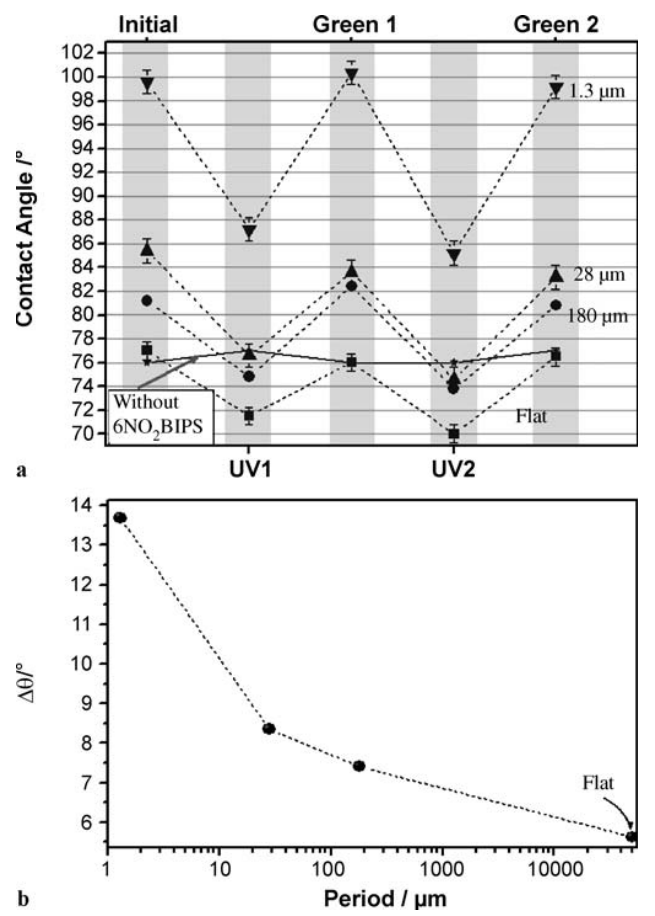

Fig. 9. (a) Mean WCA values of patterned surfaces of $10 \%$ wt SP in PEMMA for each pattern, for the flat surface and for the PEMMA surface. The media is taken after studying 10 samples of each case. (b) WCA difference $(\Delta \theta)$ from the initial one after UV irradiation. $\left(\lambda_{\mathrm{UV}}=308 \mathrm{~nm}, \mathrm{~F}_{\mathrm{UV}}=20 \mathrm{~mJ} \mathrm{~cm}{ }^{-2}, \lambda_{\text {green }}=532 \mathrm{~nm}, \mathrm{~F}_{\text {green }}=25 \mathrm{~mJ} \mathrm{~cm}^{-2}\right)($ Lygeraki et al 2008)

\begin{tabular}{lccc}
\hline Period & $1.3 \mu \mathrm{m}$ & $28 \mu \mathrm{m}$ & $180 \mu \mathrm{m}$ \\
\hline Initial & 0.68 & 0.88 & 0.94 \\
UV1 & 0.80 & 0.94 & 0.96 \\
Green1 & 0.66 & 0.89 & 0.91 \\
UV2 & 0.81 & 0.94 & 0.95 \\
Green2 & 0.68 & 0.91 & 0.94 \\
\hline
\end{tabular}

Table 1. Factor $f$ calculated by the Cassie-Baxter model under different irradiation conditions. 
In conclusion, at this section it is presented the possibility to create both hydrophobic and hydrophilic surfaces starting from the same photochromic polymeric sample by changing the topological parameters of its surface features using soft molding lithography. Due to the photochromic transformations taking place upon alternating UV and green irradiation, these surfaces can reversibly change their wettability. By careful control of the surface topology these changes can be fully controlled and tuned, in such a way that the surfaces can be wetted in a reversible manner.

\section{Reversible diffraction efficiency changes of photochromic polymer gratings}

\subsection{Introduction}

The photoinduced reversible volume changes of the lithographically patterned features of such materials are also used for realizing diffraction gratings, operating in the Raman-Nath regime, with controlled and reversible diffraction efficiency (DE). In particular, the alternating pulsed UV and green laser irradiation on the gratings causes the doped photochromic molecules to undergo transformations, which induce reversible dimensional changes to the samples. These volume changes cause reversible changes to the diffraction efficiency of the gratings, which is increased upon UV irradiation and decreased after irradiation with green laser light for various irradiation cycles. The experimental results are confirmed by a theoretical diffraction model. It is proved that the diffraction efficiency changes are attributed exclusively to the reversible dimensional changes of the imprinted structures (Fragouli et al 2008), and not to the refractive index changes as is the case in the majority of previous works. Specifically, most of the gratings with similar thickness as the here presented, are produced after irradiation with interfering beams that cause variation of the refractive index inside the samples mainly consisting of azobenzene polymers and liquid crystals. In these studies the switching procedure in the DE is due to the formation and deletion of the gratings (Yamamoto et al 2001, Fu et al 2005). Moreover, although the thickness of the produced gratings is very small, they exhibit higher DE than most of the volume gratings prepared in this regime (Tong et al 2005, Yamamoto et al 2001, Fu et al. 2005).

\subsection{Results and discussion}

A grating of period $4 \mu \mathrm{m}$ and thickness of ca. $240 \mathrm{~nm}$ was formed on the photochromic polymeric surface by the SM technique as described at section 2 . The experimental setup used for the study of the diffraction efficiency (DE) of the formed grating, is demonstrated in Figure 10. As shown, a continuous wave diode laser operating at $\lambda=822 \mathrm{~nm}$, with an incident angle adjusted to have the maximum intensity of the first-order diffracted line $\left(\mathrm{I}_{1}\right)$, was used as a reading beam. This wavelength was chosen in order not to be absorbed by the photochromic sample. The zero-order transmitted $\left(\mathrm{I}_{0}\right)$ and the $\mathrm{I}_{1}$ lines, are measured by two photodiodes, and were used for the calculation of the DE (equation 3).

$$
\mathrm{DE}=\mathrm{I}_{1} / \mathrm{I}_{0}
$$

The DE was calculated for three irradiation cycles, and Figure 11 shows the DE relative change in each case. Initially, the DE of the grating was ca $2.2 \%$, and upon UV laser irradiation it was increasing until it reached a final value. After two minutes in the dark in order to leave time to the system to relax, subsequent irradiation with green laser pulses 
caused the DE to recover close to its initial value. It is worth noticing that the relative changes of the DE during the first irradiation cycle exhibit big variations between the various examined samples, in contrary with the following irradiation cycles, where the changes are similar for all the examined gratings. This is mainly attributed to internal stresses of the polymer matrix, produced during the preparation of the gratings, that are released in a random way upon irradiation (Liang et al 2007). Nevertheless, at the second cycle during UV irradiation the DE increases with increasing number of pulses, until it stabilizes to an average value of approximately $7.4 \pm 3.0 \%$ with respect to its initial value. After green irradiation the DE is slowly reaching its initial value with increasing number of pulses. This behavior is repeated also at the third cycle.

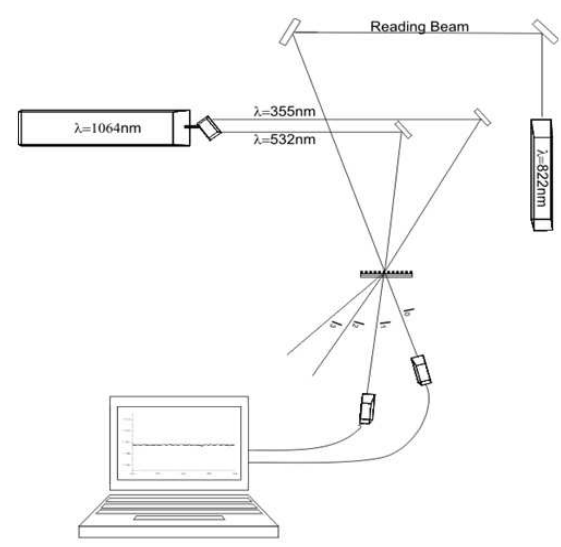

Fig. 10. Experimental setup for the measurement of the diffraction efficiency of the photochromic gratings ( $10 \%$ wt. SP in PEMMA) $\left(\lambda_{\mathrm{UV}}=355 \mathrm{~nm}, \mathrm{~F}_{\mathrm{UV}}=20 \mathrm{~mJ} \mathrm{~cm} \mathrm{c}^{-2}, \lambda_{\text {green }}=532\right.$ $\mathrm{nm}, \mathrm{F}_{\text {green }}=35 \mathrm{~mJ} \mathrm{~cm}{ }^{-2}, \lambda_{\text {reading beam }}=822 \mathrm{~nm}$.)

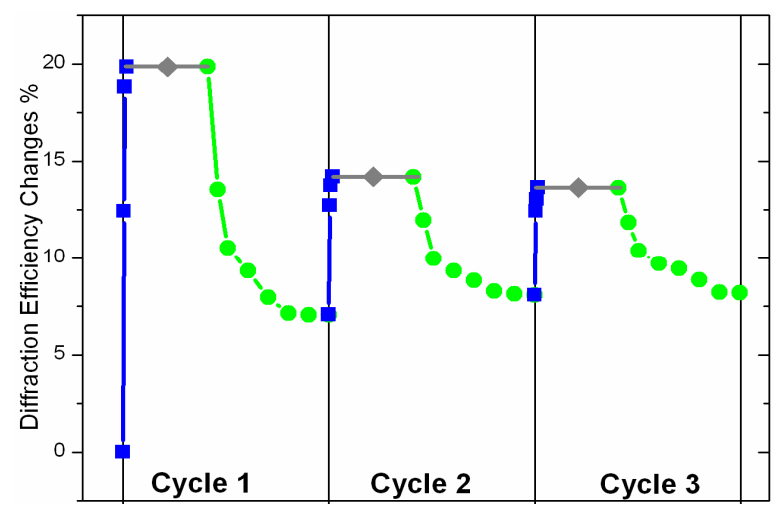

Fig. 11. Diffraction efficiency changes of the grating upon UV-green irradiation. (Fragouli et al 2008)

In order to examine the effect of the refractive index $(n)$ change of the photochromic polymer sample upon UV-green irradiation on the observed change to the DE, ellipsometric 
measurements on a similar sample were conducted. The results show that before any irradiation for $\lambda=822 \mathrm{~nm}$, the refractive index of the sample is $n=1.509$. After UV irradiation, the $n$ is higher by $\Delta n=0.029$. This difference is very small compared to the periodic refractive index variations in the grating between the photochromic polymer (1.509) and the air (1), which is actually what causes diffraction to occur. Thus it is believed that this change plays a negligible role in the measured DE relative changes. Furthermore, the small thickness of the gratings (ca. $240 \mathrm{~nm}$ ) reduces the importance of the $\Delta n$ even more.

Thus, the reversible DE changes can be attributed exclusively to the light-induced macroscopic deformations of the gratings. Specifically, Figure 12 illustrates the reversible macroscopic changes of the grating before and after UV-green irradiation as taken by AFM microscopy. As shown, the width of the stripes of the grating $(\alpha-\beta)$ is decreased by ca. $13 \%$ after UV irradiation while the distance between the two stripes $(\beta)$ is increased. A small decrease is also observed in the period of the grating $(\alpha)$ ( $\alpha$ and $\beta$ before UV, $3.971 \mu \mathrm{m}$ and $2.366 \mu \mathrm{m}$ respectively; $\alpha$ and $\beta$ after $\mathrm{UV}, 3.842 \mu \mathrm{m}$ and $2.449 \mu \mathrm{m}$ respectively). After the subsequent irradiation with green light the values recover very close to the initial ones. It is worth noticing that, as shown at Figure 12, there is a dip separating each stripe in two equal parts. As already mentioned at section 2.2, the SM technique which is followed for realizing the grating relies mainly on the capillarity that allows the viscous polymer to spontaneously fill the vertical channels that are made of the recessed features of the elastomeric mold, since the wetting lowers the overall free energy. There is always the possibility that the photochromic polymer may not fill completely such regions, thus may be mostly accumulated in the regions that are adjacent to the protruding areas of the mold, forming thus dips in the central part of the growing capillarity features. This behavior is common to different imprint lithography methods (Zankovych et al 2001, Hong and Lee 2003, Pisignano et al 2004). However, this dip is useful for the AFM morphological analysis of the patterned surfaces, since it makes the volume changes upon UV-green irradiation cycles much clearer. Moreover, it is too narrow to give any contribution to the diffracted light from the grating. In order to compare the experimental result with the existing theory, the basic equation that describes the intensity distribution of monochromatic light passing through a grating, was used (equation 5). (Born and Wolf 1999)

$$
\frac{I}{I_{0}}=\left[\frac{\sin (\pi \beta p / \lambda)}{\pi \beta p / \lambda}\right]^{2}\left[\frac{\sin (N \pi \alpha p / \lambda)}{\sin (\pi \alpha p / \lambda)}\right]^{2}
$$

I and $\mathrm{I}_{0}$ are the intensities of the light after the grating at various orders of diffraction and at zero order respectively, $\beta$ is the distance between two successive stripes, $\alpha$ is the period of the grating, $\mathrm{N}$ is the number of stripes, $\lambda$ is the wavelength of the reading beam, and $\mathrm{p}=$ $\sin \theta-\sin \theta_{0}=\mathrm{m} \lambda / \alpha(\mathrm{m}=0, \pm 1, \pm 2$ etc $)$ where $\theta_{0}$ is the angle of incidence and $\theta$ the angle of diffraction. The number of the stripes covered by the reading beam was calculated by dividing the diameter of the spot of the beam by the period of the grating in each case. The angle of incidence of the reading beam was $\theta_{0}=20^{\circ}$. In each case, by the AFM images it was measured the different value of $\alpha$ and $\beta$ before and after UV-green irradiation. Taking into account the measured parameters by the experiment and using the equation 5, it was calculated the ratio $\mathrm{I}_{1} / \mathrm{I}_{0}$, and consequently the $\mathrm{DE}$ for the gratings before and after the UVgreen irradiation. The theoretical calculations confirm that there is an increase of the DE 
after the photoisomerization process, by $8.7 \%$. The agreement between the calculated and experimental (ca. $7.4 \pm 3.0 \%$ ) values is notable, taking into account the experimental error due to factors such as the exact value of the laser beam spot size, which leads to an approximate value of the observed stripes, the imperfections of the surface introduced during the grating formation, and so on. The theoretical calculations presented, demonstrate that the decrease of the dimensions of the stripes of the gratings and of the period, are the main parameters that define the change in the DE upon irradiation (Fragouli et al 2008).

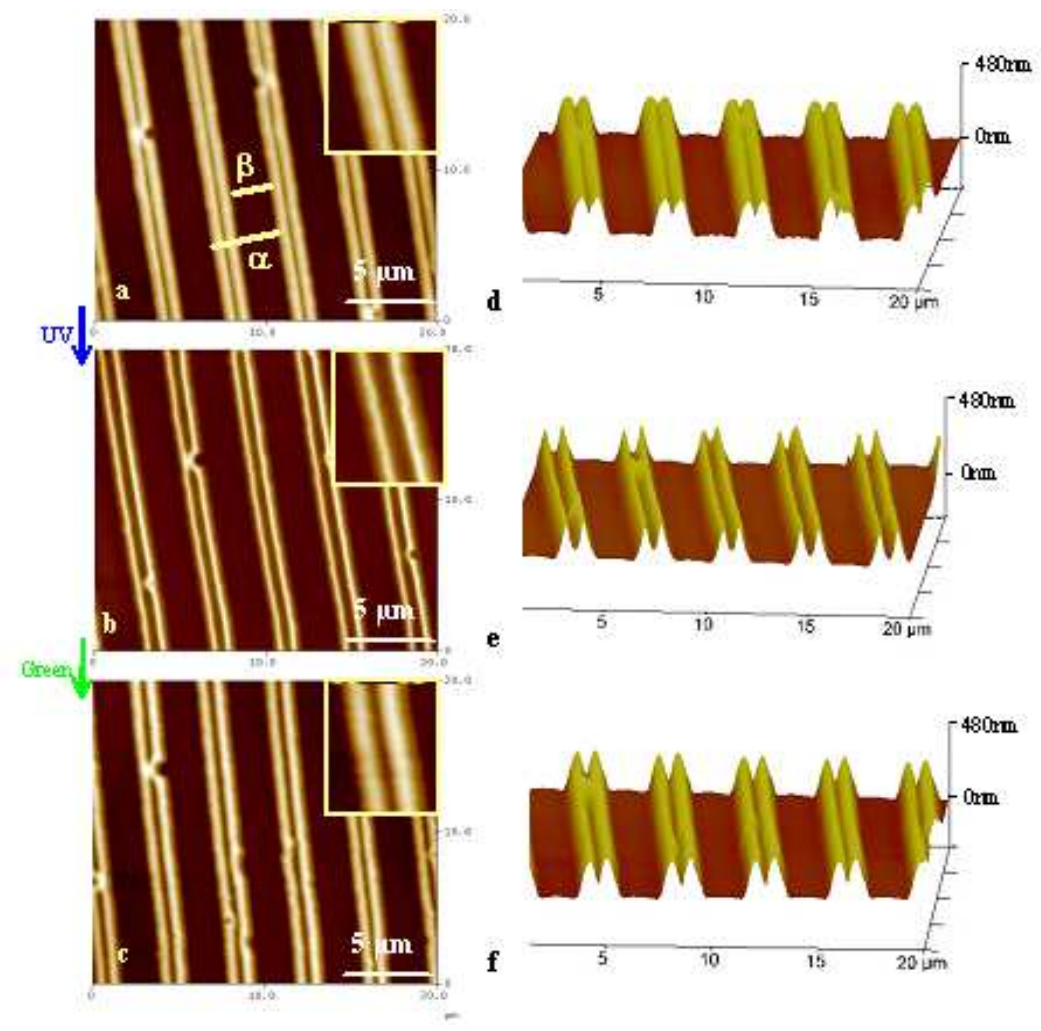

Fig. 12. AFM images of a grating before any irradiation (a) after irradiation with $20 \mathrm{UV}$ pulses (b), and after exposure to 600 green pulses (c). The insets demonstrate a single stripe in magnification. 3D images ( $d-f)$ of the grating shown in $(a-c)$, respectively. ( $\alpha$ is the period and $\beta$ is the distance between the stripes, measured at the full width at half-maximum of each feature. (Fragouli et al 2008)

\section{Conclusions}

In conclusion, it is demonstrated how soft molding lithography can be employed for the preparation of microstructured photochromic polymeric films, which undergo light controlled photomechanical changes responsible for the control of some functional characteristics of the patterned surfaces, namely the wetting properties and the diffraction 
efficiency. In particular, the light-induced isomerization of the embedded photochromic molecules in the flat surfaces is exclusively responsible for the reversible changes in their wetting properties. When the surface is microstructured by realizing patterns with the SM technique, these wetting properties are greatly enhanced. Moreover the control of the characteristics of the patterns (eg. the period), makes possible to control the light induced alterations in the wetting properties of the structured surface, demonstrating that they are influenced by both the changes in the surface polarity and the volume changes of the patterned structures. Finally, last but not least, it is demonstrated the possibility of fully manipulate the diffraction efficiency of thin photochromic polymer gratings. It is shown, that the produced gratings change their diffraction efficiency in a reversible way upon UVgreen laser irradiation. This effect, which is verified also by a theoretical diffraction model, is attributed to the reversible dimensional changes of the imprinted structures, and not to the refractive index changes as is the case in the majority of previous work. Such findings open a way for the production of optically switchable gratings based on reversible dimensional changes. Moreover, the ability to control the wettability of surfaces by microstructuring and to tune it by using photochromic molecules opens the way to the application of these optimized patterns to various microfluidic devices.

\section{Acknowledgment}

The authors would like to thank Dr. D. Pisignano, and Dr. E. Mele of the Center for Biomolecular Nanotechnologies @UNILE, Istituto Italiano di Tecnologia Via Barsanti, 73010 Arnesano (LE) and Dr. L. Persano of the National Nanotechnology Laboratory (NNL) of CNR - Istituto di Nanoscienze, via per Arnesano, 73100 Lecce, Italy for the formation of the patterned structures presented in this work.

\section{References}

Athanassiou A.; Kalyva M.; Lakiotaki K.; Georgiou S. \& Fotakis C. (2005). All-optical reversible actuation of photochromic-polymer microsystems. Advanced Materials, Vol.17, No.8, (April 2005), pp. (988-992)

Athanassiou A.; Lygeraki M. I.; Pisignano D.; Lakiotaki K.; Varda M.; Mele E.; Fotakis, C.; Cingolani R. \& Anastasiadis S. H. (2006) Photocontrolled Variations in the Wetting Capability of Photochromic Polymers Enhanced by Surface Nanostructuring. Langmuir, Vol.22, pp. (2329-2333)

Athanassiou A.; Varda M.; Mele E.; Lygeraki M. I.; Pisignano D.; Farsari M.; Fotakis, C.; Cingolani R. \& Anastasiadis S. H. (2006) Combination of microstructuring and laser-light irradiation for the reversible wettability of photosensitised polymer surfaces. Applied Physics A, Vol.83, pp. (351-356)

Athanassiou, A.; Sahinidou, D. ; Arima, V.; Georgiou, S.; Cingolani, R. \& Fotakis, C. (2006) Influence of laser wavelength and pulse duration on the degradation of polymeric films embedding photochromic molecules. Journal of Photochemistry and Photobiology A, Vol.183, pp.(182-189)

Born M. \& Wolf E. (1999) Principles of Optics: Electromagnetic theory of propagation, interference and diffraction of light, 7th Edition, Cambridge University Press. 
Brown G. H. (1971) Photochromism, Willey-Interscience, New York

Caprioli L.; Mele E.; Angilè F. E.; Girardo S.; Athanassiou A.; Camposeo A.; Cingolani R. \& Pisignano D. (2007) Photochromic spiropyran monolithic polymers: Molecular photo-controllable electroosmotic pumps for micro-fluidic devices. Applied Physics Letters, Vol.91, 113113

Cassie, A. B. D. \& Baxter, S. (1944) Wettability of porous surfaces. Transaction of the Faraday Society, Vol.40, pp.(546-551).

Fragouli, D.; Persano, L.; Paladini, G.; Pisignano, D.; Carzino, R.; Pignatelli, F.; Cingolani, R. \& Athanassiou A. (2008) Reversible Diffraction Efficiency of Photochromic Polymer Gratings Related to Photoinduced Dimensional Changes. Advanced Functional Materials, Vol.18, pp.(1617-1623).

Fu, S.; Liu, Y.; Dong, L.; Lu, Z.; Hu, W. \& Xie, M. (2005). Photo-dynamics of polarization holographic recording in spirooxazine-doped polymer films. Materials Letters, Vol.59, pp. (1449-1452)

Fu, S.; Liu, Y.; Lu, Z.; Dong, L.; Lu, Z.; Hu, W. \& Xie, M. (2005) Real-time holographic gratings recorded by $\mathrm{He}-\mathrm{Ne}$ laser in polymer films containing spirooxazine compounds pre-irradiated by UV light. Optical Materials, Vol.27, pp.(1567-1570).

Görner H. (1997). Photoprocesses in spiropyrans and their merocyanine isomers: Efects of temperature and viscosity. Chemical Physics, Vol.222, pp. (315-329)

Hong, P. S. \& Lee, H. H. (2003) Pattern uniformity control in room-temperature imprint lithography. Applied Physics Letters, Vol.83, 2441.

K. Ichimura, S.-K. Oh, M. Nakagawa, (2000) Light-Driven Motion of Liquids on a Photoresponsive Surface. Science, Vol.288, pp. (1624-1626).

Kawata, S. \& Kawata, Y. (2000). Three -dimensional optical data storage using photochromic materials. Chemical Reviews, Vol.100, No.5, pp. (1777-1788)

Liang, G.-D.; Xu, J.-T.; Fan, Z.-Q.; Mai, S.-M. \& Ryan, A. J. (2007) Effect of Substrate and Molecular Weight on the Stability of Thin Films of Semicrystalline Block Copolymers. Langmuir, Vol.23, pp. (3673-3679).

Lygeraki M. I.; Tsiranidou E.; Anastasiadis S. H.; Fotakis, C.; Pisignano D.; Cingolani R. \& Athanassiou A. (2008) Controlling the reversible wetting capability of smart photochromic-polymer surfaces by micro patterning. Applied Physics A, Vol.91, pp. $(397-401)$

Mitchell, P. (2001) (2001) Microfluidics-downsizing large-scale biology. Nature Biotechnology, Vol.19, pp. (717-721).

Patankar, N. A. (2003) On the Modeling of Hydrophobic Contact Angles on Rough Surfaces. Langmuir, Vol.19 No.4, pp (1249-1253).

Pisignano D.; Persano L.; Cingolani R.; Gigli G.; Babudri F.; Farinola G. M. \& Naso F. (2004) Soft molding lithography of conjugated polymers. Applied Physics Letters, Vol.84, No.8, (February 2004), pp. (1365-1367)

Tong, X.; Wang, G.; Yavrian, A.; Galstian, T. \& Zhao Y. (2005) Dual-mode switching of diffraction gratings based on azobenzene-polymer-stabilized liquid crystals. Advanced Materials, Vol. 17, No.3, pp. (370-374).

Walsha Z.; Scarmagnani S.; Benito-López F.; Abele S.; Nie F.-Q.; Slater C.; Byrne R., Diamond D.; Paull B. \& Macka M. (2010) Photochromic spiropyran monolithic 
polymers: Molecular photo-controllable electroosmotic pumps for micro-fluidic devices. (2010) Sensors and Actuators B, Vol.148, pp. (569-576)

Wenzel, R. N. (1936) Resistance of solid surfaces to wetting by water. Industrial and Engineering Chemistry, Vol.28, No.8, pp.(988-994).

Yamamoto, T.; Ohashi, A.; Yoneyama, S.; Hasegawa, M.; Tsutsumi, O.; Kanazawa, A.; Shiono, T. \& Ikeda, T. (2001) Phase-Type Gratings Formed by Photochemical Phase Transition of Polymer Azobenzene Liquid Crystal. 2. Rapid Switching of Diffraction Beams in Thin Films. Journal of Physical Chemistry B, Vol.105, pp. (23082313).

Yu Y.; Nakano M. \& Ikeda T. (2003). Directed bending of a polymer film by light. Nature, Vol.425, (September 2003), pp. 145

Zankovych, S.; Hoffmann, T.; Seekamp, J.; Bruch, J.-U. \& Sotomayor Torres C. M. (2001) Nanotechnology, Vol.12, 91. Nanoimprint lithography: challenges and prospects. 


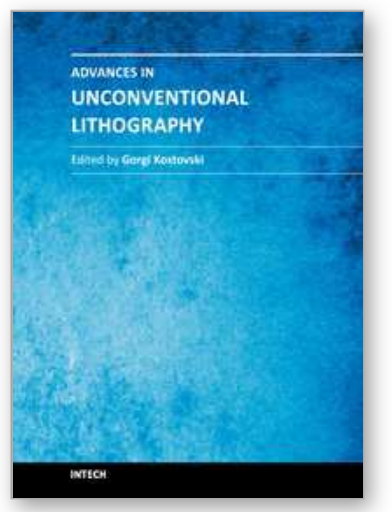

\author{
Advances in Unconventional Lithography \\ Edited by Dr. Gorgi Kostovski
}

ISBN 978-953-307-607-2

Hard cover, 186 pages

Publisher InTech

Published online 09, November, 2011

Published in print edition November, 2011

The term Lithography encompasses a range of contemporary technologies for micro and nano scale fabrication. Originally driven by the evolution of the semiconductor industry, lithography has grown from its optical origins to demonstrate increasingly fine resolution and to permeate fields as diverse as photonics and biology. Today, greater flexibility and affordability are demanded from lithography more than ever before. Diverse needs across many disciplines have produced a multitude of innovative new lithography techniques. This book, which is the final instalment in a series of three, provides a compelling overview of some of the recent advances in lithography, as recounted by the researchers themselves. Topics discussed include nanoimprinting for plasmonic biosensing, soft lithography for neurobiology and stem cell differentiation, colloidal substrates for two-tier self-assembled nanostructures, tuneable diffractive elements using photochromic polymers, and extreme-UV lithography.

\title{
How to reference
}

In order to correctly reference this scholarly work, feel free to copy and paste the following:

Despina Fragouli, Roberto Cingolani and Athanassia Athanassiou (2011). Photocontrolled Reversible Dimensional Changes of Microstructured Photochromic Polymers, Advances in Unconventional Lithography, Dr. Gorgi Kostovski (Ed.), ISBN: 978-953-307-607-2, InTech, Available from:

http://www.intechopen.com/books/advances-in-unconventional-lithography/photocontrolled-reversibledimensional-changes-of-microstructured-photochromic-polymers

\section{INTECH}

open science | open minds

\section{InTech Europe}

University Campus STeP Ri

Slavka Krautzeka 83/A

51000 Rijeka, Croatia

Phone: +385 (51) 770447

Fax: +385 (51) 686166

www.intechopen.com

\section{InTech China}

Unit 405, Office Block, Hotel Equatorial Shanghai

No.65, Yan An Road (West), Shanghai, 200040, China

中国上海市延安西路65号上海国际贵都大饭店办公楼 405 单元

Phone: +86-21-62489820

Fax: $+86-21-62489821$ 
(C) 2011 The Author(s). Licensee IntechOpen. This is an open access article distributed under the terms of the Creative Commons Attribution 3.0 License, which permits unrestricted use, distribution, and reproduction in any medium, provided the original work is properly cited. 